Article

\title{
An Instantaneous Correlation Coefficient and Simplified Coherent Averaging Method for Single-Channel Foetal ECG Extraction
}

\author{
Miao Zhang and Guo Wei * \\ School of Electrical Engineering and Automation, Harbin Institute of Technology, Harbin 150001, China; \\ zhangmiao8371@126.com \\ * Correspondence: hitweiguo@hit.edu.cn
}

Received: 21 July 2020; Accepted: 11 August 2020; Published: 14 August 2020

\begin{abstract}
In this paper, an instantaneous correlation coefficient and simplified coherent averaging method for single-channel foetal ECG (FECG) extraction is proposed. The instantaneous correlation coefficient is used to determine the position of the R peak of the measured ECG signal, and the simplified coherent averaging method is used to extract the main information of the ECG signal. The loss of the nonlinear and nonstationary characteristics by coherent averaging is recovered by threshold processing of the residual signal. The FECG signal extraction is performed in three steps. In the first step, the main information of the maternal electrocardiogram (MECG) is extracted from the abdomen electrocardiogram (AECG) signal by means of the instantaneous correlation coefficient and simplified coherent averaging method, and then the noisy FECG is obtained by subtracting the MECG obtained by simplified coherent averaging from the AECG. The second step is to extract the main information of the FECG by applying the instantaneous correlation coefficient and simplified coherent averaging method to the noisy FECG. The remaining signal is obtained by subtracting the simplified coherent averaging FECG from the noisy FECG. Thirdly, the threshold method is utilised to remove MECG residual noise and random gross value noise from the remaining signal to extract the nonlinear and nonstationary information, and the final FECG extraction is obtained by adding the nonlinear and nonstationary information to the simplified coherent averaging FECG. The validity of the proposed method is verified by experiments using synthetic data and real database data. FECG extracted by the method has the advantages of clear QRS complex wave, reasonable enhancement of $\mathrm{P}$ wave and $\mathrm{T}$ wave morphology, and no loss of nonlinear and nonstationary characteristics.
\end{abstract}

Keywords: correlation coefficient; coherent averaging; R wave detection; FECG extraction; nonstationary; nonlinear

\section{Introduction}

An electrocardiogram (ECG) is a record of physiological electrical signals in the human heart. ECG variation implies pathological changes of the heart, which cannot be indicated by other devices such as ultrasound and magnetic resonance. The foetal heart, in particular, cannot be detected by ultrasound or magnetic resonance imaging devices without full development, but foetal heart electricity is a good indicator of foetal heart development. Since 1906, when Cremer [1] first successfully detected a foetal electrocardiogram (FECG), many research achievements in FECG monitoring have been successively proposed. The research contents include various electrode development and electrode placement schemes, as well as FECG acquisition methods. FECGs are obtained in two ways: invasive scalp measurement and noninvasive abdominal skin measurement. For invasive measurements, the electrode can be attached to the scalp of a foetus while the cervix dilates during 
delivery. This approach provides a clear FECG, but the drawback is that it cannot be monitored for long periods of time at all stages of a mother's pregnancy. In contrast, noninvasive measurements are made by placing electrodes on the abdomen and chest of a pregnant woman and can be monitored at all stages of pregnancy [2,3]. However, what follows is that the measurement records contain a variety of noises, such as power line interference, movement artefacts and baseline wandering caused by respiratory movement and electrode movement, myoelectric noise caused by maternal muscle tension, electrode contact noise caused by poor electrode contact with the skin, etc. Besides this, they also inevitably contain a maternal electrocardiogram (MECG), with amplitude much higher than that of the FECG $[4,5]$. The power line interference is usually a $50 \mathrm{~Hz}($ or $60 \mathrm{~Hz})$ signal, and a notch device is adopted to eliminate it. The processing of FECG signals falls into two categories. One is to obtain the R peaks of the FECG by analysing and calculating the foetal heart rate (FHR) or heart rate variability (HRV). The other is to extract the pure FECG morphological waveform from the abdominal mixed signals, because the waveform contains information that could reflect more abundant FECG information used to diagnose foetal heart physiological characteristics.

FECG extraction can be divided into multichannel and single-channel methods. The multichannel approach can take advantage of information redundancy in multichannel signals, so the FECG can be more clearly extracted by "correcting" the inaccurate signals collected by individual leads. However, multichannel extraction methods are more suitable for in-hospital detection due to the large number of electrodes, which is inconvenient for use in portable family equipment and increases the complexity and power consumption of the equipment. Single-channel methods require only one measuring electrode, which can reduce equipment consumption and improve wearing comfort. Nevertheless, without other information for "corroboration" or correction, single-channel extraction is vulnerable to interference. It is often necessary to analyse characteristics of the MECG and FECG to obtain corresponding prior or posterior information, so as to establish a basic function model to extract the FECG. At present, the single-channel methods include the template subtraction (TS) method [6-10], adaptive filtering [11-13], wavelet transform, Wigner-Ville distribution [14-17], S-transform [18], sequential total variation [19], adaptive neuro-fuzzy reasoning system, extended Kalman Filtering [20], etc. However, unreasonable ECG modeling or selection of a different base function may affect FECG extraction results, which often leads to distortion of extraction and loss of nonlinear and nonstationary characteristics. Moreover, these single-channel extraction methods mostly obtain noisy FECGs requiring further denoising or enhancement processing. Due to the complexity of the physiological activities and pathological changes of the human heart, up to now, no wavelet function for all ECG signals or universal ECG model has been developed. Neural network learning methods need a large amount of data for training, but the target signals are difficult to obtain. Therefore, they are not suitable for ECG signal extraction. In addition to the above single-channel methods, multichannel methods can also be used to extract FECGs after transforming the single-channel signal to multichannel signals, such as pseudo-phase space reconstruction combined with the singular value decomposition (SVD) [21], principal component analysis (PCA) [22], and independent component analysis (ICA) extraction methods [23,24]. Pseudo-phase space analysis is appropriate for stationary signals, but for nonstationary ECG signals, it reduces nonstationarity. Blind source separation methods such as ICA in pseudo-phase space are not effective and often fail to extract.

In this paper, an instantaneous correlation coefficient and a simplified coherent averaging method for single-channel foetal ECG extraction are proposed. An instantaneous correlation coefficient method based on the ECG reference model is proposed to improve the accuracy of ECG R peak detection. The MECG and FECG mean waveforms are extracted step by step by the simplified coherent averaging method, and the nonlinear, nonstationary characteristics of the FECG are recovered from the residual signal by the threshold method. The validity of the proposed method was verified by experiments using synthetic signals and real ECG data from the database for the identification of systems (DaISy), abdominal and direct fetal ECG database (ADFECGDB), and non-invasive fetal ECG database (NIFECGDB). 


\section{Methods}

\subsection{Instantaneous Correlation Coefficient Method for Detecting $R$ Peaks}

The key technique of the coherent averaging method is to determine the R peak position of the ECG. The correlation coefficient is an effective index to reflect the similarity of two signals, and the correlation coefficient is the largest when the beats of two ECGs are overlapped. In this paper, a dimensionless heart beat model was first established. The model was scaled according to the heart rate cycle and standard deviation of the ECG signals to establish the reference heartbeat, and the windowing correlation coefficient between the reference heart beat and ECG signals was calculated. The width of the added time window is the heart rate cycle of the reference heartbeat, and the R peak of the reference heart beat is placed at the midpoint of the time window. When the reference beat moves with the window, the correlation coefficient is instantaneous. When the $\mathrm{R}$ peak of the reference heartbeat passes the $\mathrm{R}$ peak of the ECG signal, the instantaneous correlation coefficient reaches its maximum value.

\subsubsection{Dimensionless Heart Beat Model}

The ECG waveform and the normal range of the parameters were obtained from the literature [4,25]. Heart rate (HR): 60-100 bpm. P wave amplitude: $0.25 \pm 0.05 \mathrm{mv}$. Interval: $110 \pm 20 \mathrm{~ms}$. QRS complex amplitude: $1.6 \pm 0.5 \mathrm{mv}$. Interval: $100 \pm 20 \mathrm{~ms}$. $\mathrm{R}$ wave amplitude: $1.6 \pm 0.5 \mathrm{mv}$. $\mathrm{Q}$ wave amplitude: $0.25 \times \mathrm{R}$ wave. $\mathrm{T}$ wave amplitude: $0.1-0.5 \mathrm{mv}$. Interval: $160 \mathrm{~ms}$. According to the normal range of a heart beat, the reference heart beat model was established by taking the middle value. The dimensionless $\mathrm{R}$ peak and heart rate cycle were adjusted to obtain a dimensionless beat model, as shown in Figure 1.

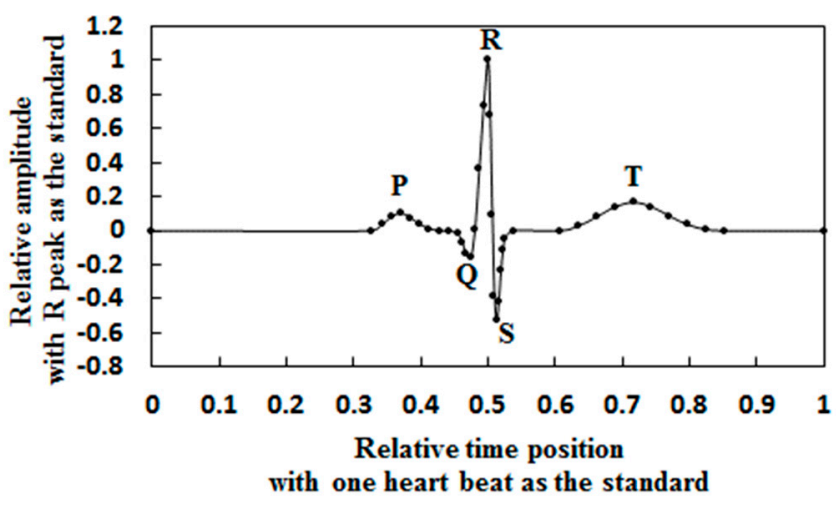

Figure 1. Dimensionless heart beat model.

\subsubsection{Instantaneous Correlation Coefficient}

Let $x(n)$ be the measured ECG signal, with sampling frequency $f_{s}$, signal length $N$, and reference heart rate $H R$. We scale the dimensionless heart beat model according to $H R$ and resample according to $f_{s}$, with $h(k)$ denoting the reference heart beat, where $k=1,2, \cdots, K$. $K$ is the reference heart beat length.

$$
K=60 f_{s} / H R
$$

Firstly, the baseline wandering of $x(n)$ is removed and the high-frequency noise is pre-denoised; then, the instantaneous correlation coefficient $R_{x}(n)$ is calculated.

$$
R_{x}(n)=\frac{\sum_{k=1}^{K} x(n-K / 2+k) h(k)}{\sqrt{\sum_{k=1}^{K}(x(n-K / 2+k))^{2} \sum_{k=1}^{K}(h(k))^{2}}}
$$


Here, $n=1,2, \cdots, N$. When $n-K / 2+k$ is smaller than 1 or larger than $N, x(n-K / 2+k)$ is equal to 0 .

\subsubsection{R Peak Detection Based on the Instantaneous Correlation Coefficient}

The maximum value of $R_{x}(n)$ is obtained at the $\mathrm{R}$ peak position of $x(n)$. The detection of the $\mathrm{R}$ peak is realised by the detection of $R_{x}(n)$ peaks. Since the $\mathrm{R}$ peak value of the reference heat beat $h(k)$ established in this paper is positive, if the $\mathrm{R}$ peak value of $x(n)$ is positive, there is a maximum value of $R_{x}(n)$ at the position of the $\mathrm{R}$ peak value of $x(n)$. On the contrary, if the $\mathrm{R}$ peak of $x(n)$ is negative, there is a minimum value of $R_{x}(n)$ at the $\mathrm{R}$ peak of $x(n)$. Therefore, $\mathrm{R}$ peak detection needs to first determine the direction of the $\mathrm{R}$ peak.

(1) Determining the R Peak Threshold and R Peak Sequence

Firstly, we calculate the extreme point sequence $A_{R}(k)$ of $R_{x}(n)$ (including the maxima and minima) and the corresponding time position sequence $T_{R}(k)$.

$$
\begin{aligned}
& A_{R}(k)=\left\{\begin{array}{lc}
R_{x}(n) & \left|R_{x}(n-1)\right|<\left|R_{x}(n)\right|>\left|R_{x}(n+1)\right| \\
\text { neglect } & \text { otherwise }
\end{array}\right. \\
& T_{R}(k)=\left\{\begin{array}{lc}
n & \left|R_{x}(n-1)\right|<\left|R_{x}(n)\right|>\left|R_{x}(n+1)\right| \\
\text { neglect } & \text { otherwise }
\end{array}\right.
\end{aligned}
$$

We calculate the mean and standard deviation of the absolute value of $A_{R}(k)$. The R peak threshold $R$ th is determined by the following formula:

$$
R \operatorname{th}=\operatorname{mean}\left(\left|A_{R}(k)\right|\right)+\operatorname{stdev}\left(\left|A_{R}(k)\right|\right)
$$

where mean $(\cdot)$ represents the mean value function and stdev $(\cdot)$ represents the standard deviation function.

The maximum value greater than threshold $R$ th in $A_{R}(k)$ is denoted $P_{u p}\left(k_{1}\right)$, and the corresponding time position is $T_{\text {up }}\left(k_{1}\right)$. The minimum value less than $-R t h$ is denoted $P_{\text {low }}\left(k_{2}\right)$, and the corresponding time is $T_{\text {low }}\left(k_{2}\right)$. The respective average values of $P_{u p}$ and $P_{\text {low }}$ are calculated. The larger absolute value of the average value is the $\mathrm{R}$ peak sequence, denoted $R_{\text {peak }}(k)$, and the corresponding time sequence is $T_{\text {peak }}(k)$, i.e.,

$$
\begin{aligned}
& R_{\text {peak }}(k)= \begin{cases}P_{\text {up }}\left(k_{1}\right) & \text { if } \overline{\left|P_{\text {up }}\right|}>\overline{\left|P_{\text {low }}\right|} \\
-P_{\text {low }}\left(k_{2}\right) & \text { if } \overline{\left|P_{\text {up }}\right|}<\overline{\left|P_{\text {low }}\right|}\end{cases} \\
& T_{\text {peak }}(k)=\left\{\begin{array}{ll}
T_{\text {up }}\left(k_{1}\right) & \text { if } \overline{\left|P_{\text {up }}\right|}>\overline{\left|P_{\text {low }}\right|} \\
T_{\text {low }}\left(k_{2}\right) & \text { if } \overline{\left|P_{\text {up }}\right|}<\overline{\left|P_{\text {low }}\right|}
\end{array} .\right.
\end{aligned}
$$

(2) Eliminating Wrong R Peaks

There may be non-R peak data in $R_{\text {peak }}(k)$, which need to be discriminated and eliminated. First, the RR interval $T_{R R}(k)$ and mean RR interval $\overline{T_{R R}(k)}$ are calculated for $R_{\text {peak }}(k)$ :

$$
\begin{gathered}
T_{R R}(k)=T_{\text {peak }}(k+1)-T_{\text {peak }}(k) \\
\overline{T_{R R}(k)}=\frac{1}{K-1} \sum_{k=1}^{K-1} T_{\text {peak }}(k)
\end{gathered}
$$

where $K$ is the cardinality of $R_{\text {peak }}(k)$.

$T_{R R}(k)<0.5 \overline{T_{R R}}$ indicates that there is a wrong $R$ peak between $R_{\text {peak }}(k)$ and $R_{\text {peak }}(k+1)$ that needs to be removed. When $T_{R R}(k)=(0.2 \sim 0.5) \overline{T_{R R}(k)}$, if $T_{R R}(k-1)>\overline{T_{R R}(k)}, R_{\text {peak }}(k+1)$ is eliminated, 
and if $T_{R R}(k-1)+T_{R R}(k)<\overline{T_{R R}(k)}, R_{\text {peak }}(k)$ is eliminated. In addition, the smaller absolute value of $R_{\text {peak }}(k)$ and $R_{\text {peak }}(k+1)$ is eliminated.

After eliminating the wrong $\mathrm{R}$ peak, we recalculate $T_{R R}(k)$ and $\overline{T_{R R}(k)}$. We repeat the above steps until there is no wrong $R$ peak to be eliminated.

\subsection{Simplified Coherent Averaging Method}

The coherent averaging method is an effective way to extract periodic signals. An ECG is a typical nonlinear, nonstationary, quasi-periodic signal. Although different beats have different lengths, they have temporal correlation. The PQRST waves of different beats are similar. Therefore, coherent averaging can be used to denoise and extract ECG signals.

\subsubsection{Coherent Averaging Method}

Let $x(t)$ be the ECG observation signal, $s(t)$ be the ECG original signal, and $n(t)$ be the noise.

$$
x(t)=s(t)+n(t)
$$

We take the R peak of the ECG as the interception point and the RR interval as the length. $x(t)$ is divided into $M-1$ segments, denoted $x_{i}(t)$ :

$$
x_{i}(t)=s_{i}(t)+n_{i}(t), \quad i=0,1, \cdots, M-1
$$

where $M$ is the number of $\mathrm{R}$ peaks in $x(t)$.

Due to the nonstationarity of ECG signals, the lengths of the RR intervals are not equal. Therefore, $x_{i}(t)$ needs to be linearly scaled to the same length and resampled to the same sample number. Then, the resampled $x_{i}(t)$ is used as a row vector to construct a matrix, and coherent averaging is obtained by calculating the column average of the matrix:

$$
\begin{gathered}
\bar{x}(t)=\frac{1}{M-1} \sum_{i=1}^{M-1} x_{i}(t)=\frac{1}{M-1} \sum_{i=1}^{M-1}\left(s_{i}(t)+n_{i}(t)\right) \\
\bar{x}(t)=\bar{s}(t)+\frac{1}{M-1} \sum_{i=1}^{M-1} n_{i}(t)
\end{gathered}
$$

where $\bar{s}(t)=\frac{1}{M-1} \sum_{i=1}^{M-1} s_{i}(t)$.

According to Formula (13), the second term is the coherent averaging noise, which is only $1 /(M-1)$ times as much as the original noise. If $M$ is large enough, $\bar{x}(t) \approx \bar{s}(t)$. We reversely scale and resample $\bar{x}(t)$ back to the original length and place it repeatedly according to the initial position. Thus, the denoised signal is obtained.

The coherent averaging method can obviously suppress the noise and enhance the local information of PQRST waves in the ECG. However, the nonlinear and nonstationary characteristics are weakened at the same time; in particular, the difference in amplitude cannot be recovered.

\subsubsection{Simplified Coherent Averaging Method}

Although the coherent averaging method has its shortcomings, it can be used to extract an FECG from mixed abdomen ECG (AECG) signals. It is not used for extracting a perfect MECG, but for eliminating the MECG from the signal, so that the FECG can be revealed in the remaining signal. Assuming that the ECG signal is stationary for a short time and that the change of QRS width with instantaneous beats can be ignored, the simplified coherent averaging method is established as follows: 
(1) Determine the R peak position of the MECG in the AECG signal and the corresponding RR intervals.

(2) Take the $R$ peak position as the midpoint, and take the maximum RR interval as the length. The data beyond the AECG signal are filled with zeroes.

(3) Form the heart beat matrix with the truncated data segment. Calculate the column mean value.

(4) Place the mean value repeatedly according to the R peak positions calculated in Step 1. Abandon the overlapped parts to obtain the coherent averaging MECG signal.

When the MECG is extracted by the conventional coherent averaging method, the time domain is linearly scaled. As a result, the nonstationarity of time is restored to some extent, while the waveform amplitude only retains the average result, so the amplitude deviation caused by the nonstationarity is not recovered. However, the simplified coherence averaging method in this paper only extracts the stationary information of the MECG, and the nonstationary information is left in the remaining signals. Therefore, the useful information of the FECG will not be lost when the coherent averaging MECG is eliminated. Even if the QRS complexes of the FECG and MECG overlap, the information of the FECG will not be lost.

\subsection{Extracting a Noisy FECG}

In contrast to common ECG signal denoising, for extracting an FECG, there are not only various noises, but also the MECG existing in the AECG. That is, there are two quasi-periodic signals in the signal, which makes it difficult to extract the FECG.

However, commonly, the FECG heart rate is between 120 and $180 \mathrm{bpm}$, and the MECG heart rate is between 60 and $100 \mathrm{bpm}$. The amplitude of the MECG is 5-10 times larger than that of the FECG [25-27]. Therefore, we think about the denoising in another way: For the MECG, the FECG can be treated as noise, and the MECG can be firstly extracted from the AECG based on the proposed method. Then, the MECG is subtracted from the AECG to get the FECG only mixed with noise, i.e., a noisy FECG.

Before the MECG is extracted by the proposed method, the AECG is pretreated to eliminate power line interference and baseline drift. When calculating the instantaneous correlation coefficient, the high-frequency noise is properly filtered under the premise of ensuring the QRS shape.

The extraction process of a noisy FECG is as follows:

(1) Remove power line interference and baseline wandering in the AECG.

(2) Determine the reference heart rate of $M E C G$ using $A E C G$, and reconstruct the reference heart beat of the MECG using the dimensionless heart beat model.

(3) Calculate the instantaneous correlation coefficient $R_{A}(n)$.

(4) Detect the $\mathrm{R}$ peak position of the MECG using $R_{A}(n)$.

(5) Extract the MECG using the simplified coherence average method and denote it by $M E C G_{c}$.

(6) Obtain a noisy FECG by subtracting $M E C G_{c}$ from the AECG and denote it by $F E C G_{n}$.

$$
\operatorname{FECG}_{n}(n)=\operatorname{AECG}(n)-\operatorname{MECG}_{c}(n)
$$

\subsection{FECG Enhancement and Recovery of Nonstationarity and Nonlinear Characteristics}

The MECG residue is included in $F E C G_{n}$ in addition to other noise, which affects the morphology of the FECG significantly and the clarity of the QRS complex, as shown in Figure 2a. In order to eliminate these residues, we continue to apply the proposed method above to extract the FECG from $F E C G_{n}$ and denote it by $F E C G_{c}$. If $F E C G_{c}$ is taken as the final extraction result (clear FECG), we will lose the nonlinear and nonstationary characteristics of the FECG. For this reason, in this paper, the MECG residue is eliminated using the threshold method after the extraction of $F E C G_{c}$ and 
then superimposed with $F E C G_{c}$ so as to enhance the FECG and recover the nonlinear, nonstationary characteristics (see Figure 2b).

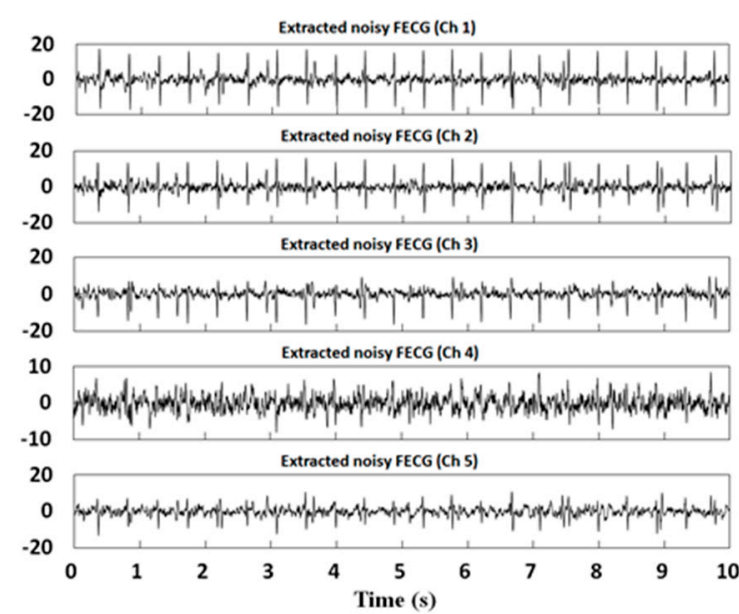

(a) Extraction results

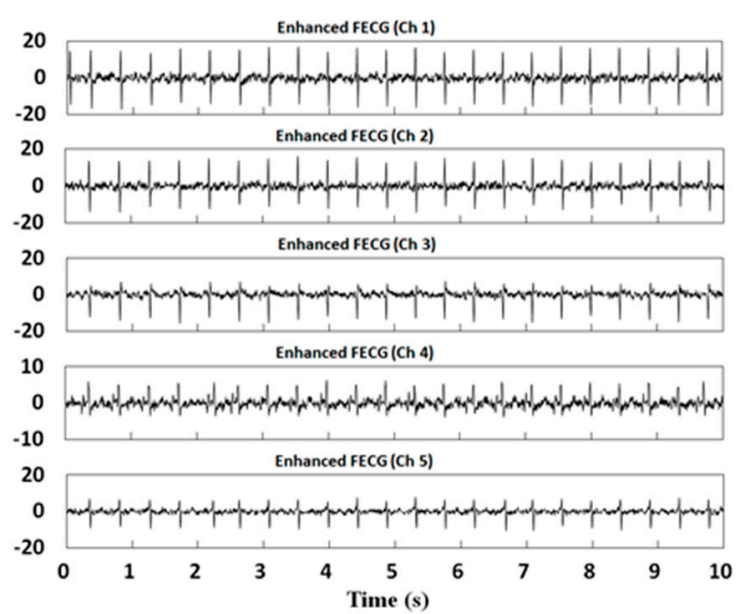

(b) Enhancement of extraction results

Figure 2. The extraction and enhancement of foetal electrocardiograms (FECGs) obtained using the proposed method for the DaISy database.

(1) Use $F E C G_{n}$ to estimate the reference heart rate of $F E C G$; the FECG reference heart beat $h(k)$ is established using the dimensionless heart beat model.

(2) Calculate the instantaneous correlation coefficient $R_{F}(n)$.

(3) Detect the $\mathrm{R}$ peak of the FECG using $R_{F}(n)$.

(4) Extract the FECG using the simplified coherent averaging method and denote it by FECG . $_{\text {. }}$

(5) Subtract $F E C G_{c}$ from $F E C G_{n}$ to obtain the remaining signal $r_{F}(n)$.

$$
r_{F}(n)=F E C G_{n}(n)-F E C G_{c}(n)
$$

(6) Calculate the standard deviation of $F E C G_{c}$ and define the threshold $T h$ :

$$
T h=C \cdot \operatorname{stdev}\left(F E C G_{c}\right)
$$

where $C$ is the ratio, which is 1 in this paper.

(7) Set the absolute value of $r_{F}(n)$ greater than $T h$ to 0.

$$
\Delta r_{F}(n)= \begin{cases}0 & \mid r_{F}(n) \\ r_{F}(n) & \left|r_{F}(n)\right| \leq T h\end{cases}
$$

(8) Recover the nonlinearity and nonstationarity of the FECG, and obtain the final extraction of the FECG.

$$
\operatorname{FECG}(n)=F E C G_{c}(n)+\Delta r_{F}(n)
$$

In the process of FECG extraction using the simplified coherent averaging method, the FECG's stationary information, nonstationary information, MECG residue, and noise are all left in the remaining signal. In order to recover the nonstationary information of the FECG, the residual signal should be processed. According to the analysis, the coherent averaging FECG represents the main component of the FECG, and the nonstationary value of amplitude is not larger than the variance of the coherent averaging FECG. Therefore, taking the variance of the coherent averaging FECG as the threshold value, useful signals can be retained by eliminating the values with absolute values larger than the threshold 
value in the remaining signal, and the nonstationary characteristics of the FECG can be finally restored by adding them with the coherent averaging FECG.

A flow chart of the method proposed in this paper is displayed in Figure 3.

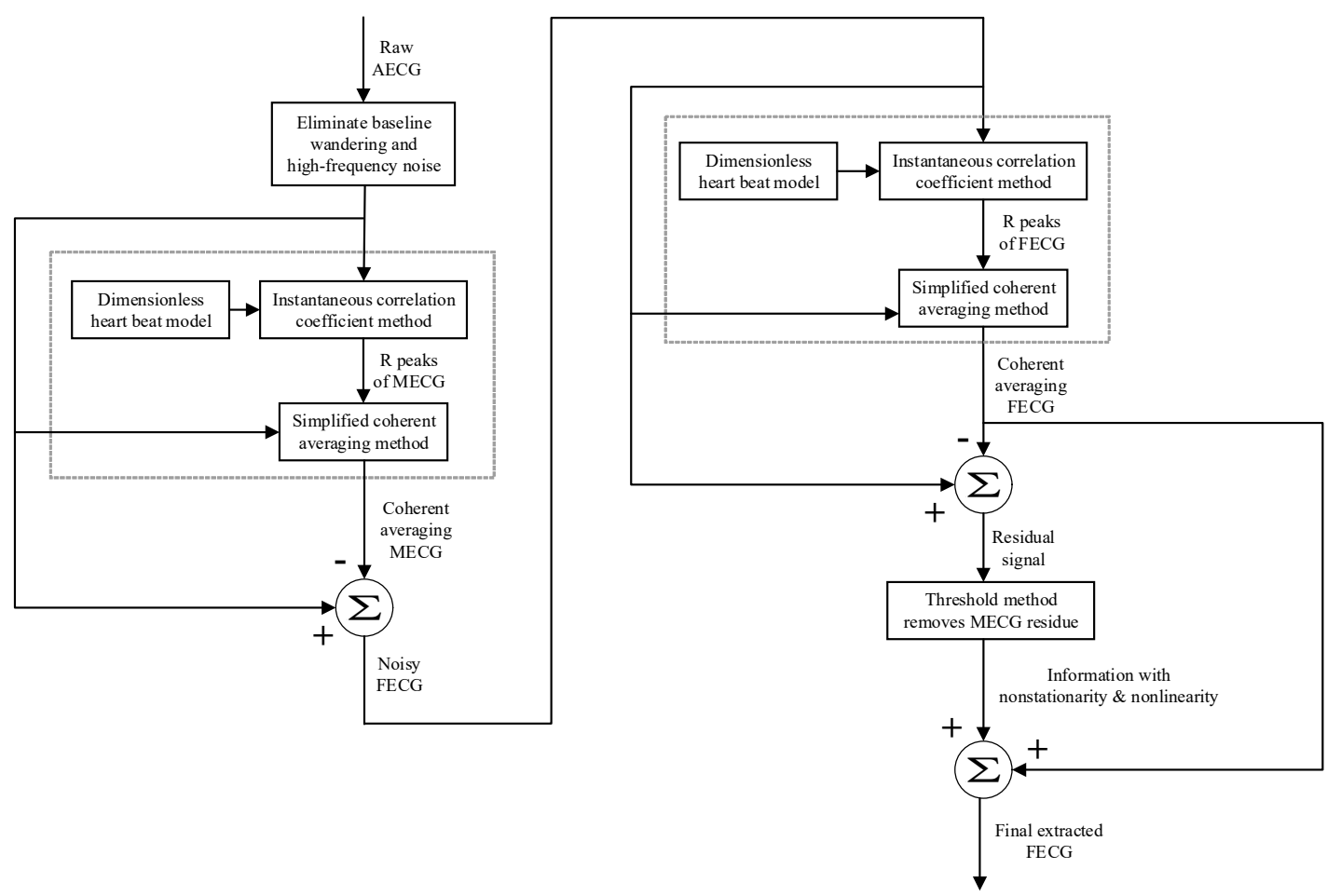

Figure 3. Flow chart of the proposed method. AECG is abdominal ECG and MECG is maternal ECG.

\section{Experimental Data and Evaluation of Results}

\subsection{Source of Experimental Data}

In order to verify the performance of the proposed method, synthetic data and real data were used for experiments.

\subsubsection{Synthetic Data}

In the literature [28], a standard heart model based on a single dipole vector was utilised to simulate MECGs and FECGs. Gaussian white noise was added to synthesise an abdominal signal. The open source toolkit FECGSYN $[29,30]$ in the Open-Source Electrophysiological Toolbox (OSET) can also be used to simulate MECGs, FECGs, or non-invasive FECGs. Although the toolkit can be used to simulate signals of any parameters, it cannot fully simulate the nonlinear and nonstationary characteristics of the complex human body system. In this paper, we chose existing real databases to provide the basic data, such as abdominal and direct fetal ECG database (ADFECGDB) [31], non-invasive fetal ECG database (NIFECGDB) [32], database for the identification of systems (DaISy) [33], and HIT-BIH arrhythmia database (MIT-BIH) [34]. The direct scalp FECGs, thoracic MECGs and adult ECGs are preprocessed by filtering and eliminating baseline wandering before they can be used for synthesising abdominal signals. Table 1 lists the signals selected for this paper. Figure 4 shows the processed results of the direct scalp FECGs in the ADFECGDB database. The red line represents the original scalp FECG and the black line represents the processed FECG. The FECGs, after being preprocessed, should have complete QRS waveforms and accurate positions and amplitudes of $R$ peaks, with smooth waveforms and clear $\mathrm{P}$ and $\mathrm{T}$ waves. In addition, the sampling frequency of ECG signals from the DaISy and MIT-BIH databases was different to that from ADFECGDB and NIFECGDB-250 and $360 \mathrm{~Hz}$, respectively. For data synthesis, the sampling frequency should be uniformly increased to 
$1 \mathrm{kHz}$. Figure 5 shows a comparison of the waveform and spectrum before and after resampling of $\mathrm{Ch} 8$ in the DaISy database. Figure 6 shows the waveform and spectrum comparison before and after resampling of a103 in the MIT-BIH database. It can be seen that the waveform and the spectrum are consistent before and after resampling.

Table 1. FECGs and MECGs used for synthesising signals.

\begin{tabular}{|c|c|c|c|c|c|c|c|}
\hline $\begin{array}{l}\text { ECG } \\
\text { Type }\end{array}$ & Database & $\begin{array}{l}\text { Electrode } \\
\text { Position }\end{array}$ & $\begin{array}{l}\text { Number } \\
\text { and } \\
\text { Channel }\end{array}$ & $\begin{array}{l}\text { Average } \\
\text { Heart Rate } \\
\text { (bpm) }\end{array}$ & $\begin{array}{l}\text { Sampling } \\
\text { Rate (Hz) }\end{array}$ & $\begin{array}{c}\text { Data } \\
\text { Length (s) }\end{array}$ & $\begin{array}{c}\text { Waveform } \\
\text { Similarity } \\
\left(C_{R}\right)\end{array}$ \\
\hline \multirow{5}{*}{ FECG } & \multirow{5}{*}{ ADFECGDB } & \multirow{5}{*}{ Scalp } & r01 & 128 & \multirow{5}{*}{1000} & \multirow{5}{*}{10} & 0.962 \\
\hline & & & r04 & 124 & & & 0.949 \\
\hline & & & r07 & 127 & & & 0.950 \\
\hline & & & r08 & 128 & & & 0.981 \\
\hline & & & r10 & 117 & & & 0.919 \\
\hline \multirow{11}{*}{ MECG } & \multirow{3}{*}{ DaISy } & \multirow{3}{*}{ Chest } & Ch6 & 82 & \multirow{3}{*}{250} & \multirow{3}{*}{10} & 0.995 \\
\hline & & & Ch7 & 82 & & & 0.997 \\
\hline & & & $\mathrm{Ch} 8$ & 82 & & & 0.996 \\
\hline & \multirow{8}{*}{ NIFECGDB } & \multirow{8}{*}{ Chest } & ca473 & 89 & \multirow{8}{*}{1000} & \multirow{8}{*}{10} & 0.993 \\
\hline & & & ca585 & 89 & & & 0.992 \\
\hline & & & ca649 & 96 & & & 0.996 \\
\hline & & & ca748 & 91 & & & 0.991 \\
\hline & & & ca771 & 87 & & & 0.992 \\
\hline & & & ca840 & 85 & & & 0.992 \\
\hline & & & ca886 & 92 & & & 0.995 \\
\hline & & & ca902 & 90 & & & 0.991 \\
\hline \multirow{10}{*}{$\begin{array}{l}\text { Adult } \\
\text { ECG }\end{array}$} & \multirow{10}{*}{ MIT-BIH } & \multirow{10}{*}{ Chest } & a100 & 73 & \multirow{10}{*}{360} & \multirow{10}{*}{10} & 0.998 \\
\hline & & & a101 & 68 & & & 0.998 \\
\hline & & & a103 & 67 & & & 0.999 \\
\hline & & & a112 & 87 & & & 0.996 \\
\hline & & & a113 & 58 & & & 0.999 \\
\hline & & & a115 & 63 & & & 0.999 \\
\hline & & & a117 & 50 & & & 0.997 \\
\hline & & & a119 & 65 & & & 0.998 \\
\hline & & & a122 & 87 & & & 0.998 \\
\hline & & & a123 & 45 & & & 0.998 \\
\hline
\end{tabular}

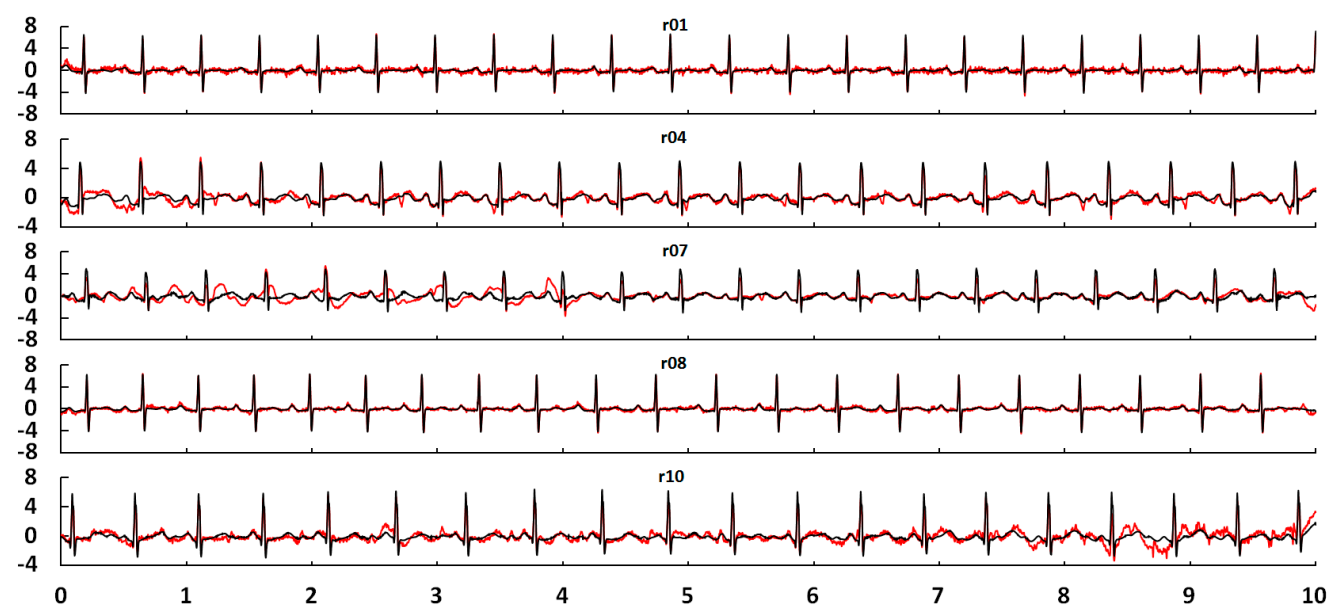

Figure 4. Direct scalp FECGs after filtering and eliminating baseline wandering in ADFECGDB. 


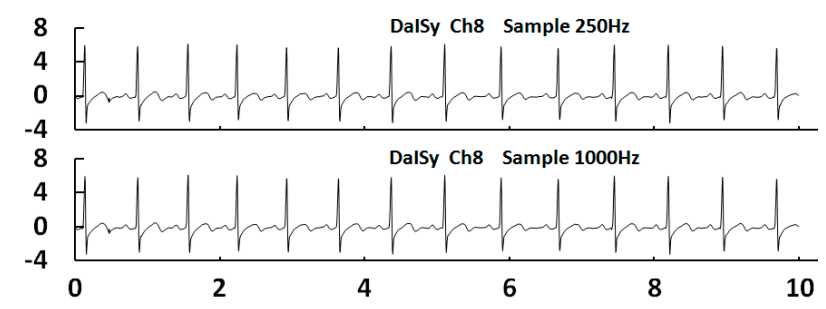

(a) Waveform comparison after resampling

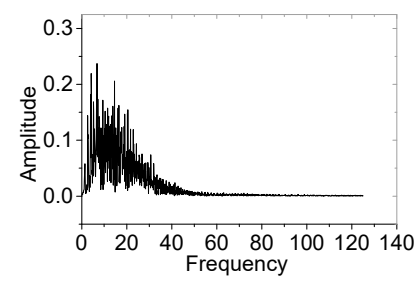

(b) Spectrum of the signal in $250 \mathrm{~Hz}$

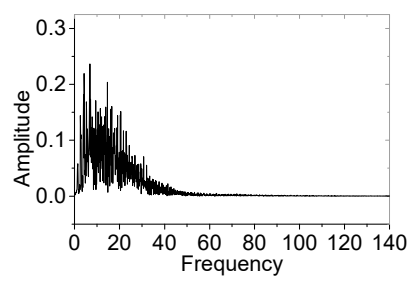

(c) Spectrum of the signal in $1000 \mathrm{~Hz}$

Figure 5. Waveform and spectrum before and after resampling of Ch8 in the DaISy database.

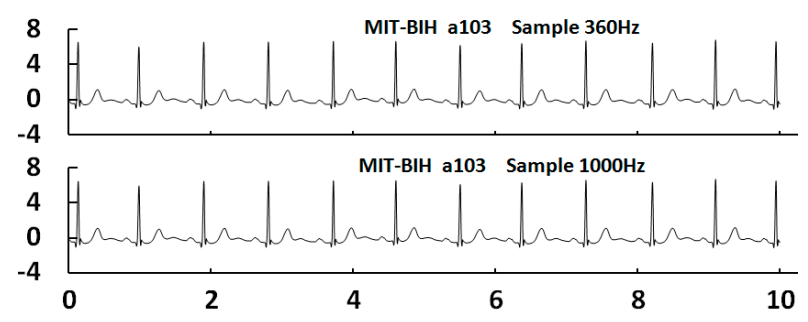

(a) Waveform comparison after resampling

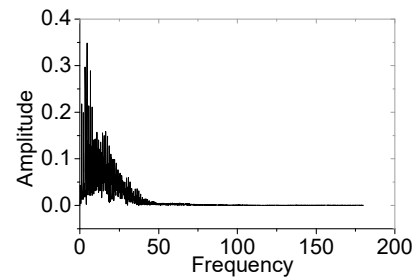

(b) Spectrum of the signal in $360 \mathrm{~Hz}$

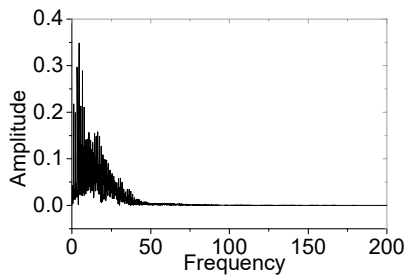

(c) Spectrum of the signal in $1000 \mathrm{~Hz}$

Figure 6. Waveform and spectrum before and after resampling of a103 in the MIT-BIH database.

\subsubsection{Real Data}

The real data for the experiments were acquired from the DaISy, ADFECGDB, and NIFECGDB databases. The DaISy database was provided by Belgian scholar Lathauwer and is a single data set containing eight channels of skin potential recordings of a pregnant woman, of which the first five channels were collected at an abdomen electrode and the last three channels were collected at a chest electrode. The sampling rate is $250 \mathrm{~Hz}$ and the time is $10 \mathrm{~s}$. The ADFECGDB database contains five data sets, namely, r01, r04, r07, r08, and r10. Each group of data contains one direct scalp measurement signal and four abdominal measurement signals. The sampling rate is $1 \mathrm{kHz}$ and the time is $5 \mathrm{~min}$. The NIFECGDB database consists of 55 multichannel abdominal FECG records, all from the same tester, between 21 and 40 weeks of gestation. Each set of recordings contains two chest signals and three or four abdomen signals. The sampling rate is $1 \mathrm{kHz}$ and the resolution is $16 \mathrm{bit}$. 


\subsection{Evaluation of Experimental Results}

The experimental results of the synthetic signals were evaluated using the signal-noise ratio (SNR) and correlation coefficient. Let $s_{f}, s_{m}$, and $n$ represent the FECG, MECG, and noise signals, respectively. $s$ represents the mixed signals in the abdomen, and $\hat{s}_{f}$ represents the estimation of $s_{f}$, that is, the FECG extraction result. Suppose that $s_{f}, s_{m}$, and $n$ are independent and that the abdomen mixed ECG signal is the superposition of the three signals:

$$
s=s_{m}+s_{f}+n
$$

If $s_{f}$ is taken as the signal of concern, then both $s_{m}$ and $n$ are considered noise. Therefore, the SNR of the mixed signal $s$ (i.e., input SNR) is defined as

$$
S N R_{i n}=10 \log _{10}\left(\frac{P_{s_{f}}}{P_{\left(s_{m}+n\right)}}\right)
$$

where $P s_{f}=\frac{1}{N} \sum_{n=1}^{N} s_{f}^{2}$ and $P_{\left(s_{m}+n\right)}=\frac{1}{N} \sum_{n=1}^{N}\left(s_{m}+n\right)^{2}$ are the energy of the FECG and the energy of the MECG plus noise.

For the successfully extracted FECG, the SNR of $\hat{s}_{f}$ (i.e., output SNR) is defined as

$$
S N R_{\text {out }}=10 \log _{10}\left(\frac{P_{s_{f}}}{P_{\left(\hat{s}_{f}-s_{f}\right)}}\right)
$$

where $P_{\left(\hat{s}_{f}-s_{f}\right)}=\frac{1}{N} \sum_{n=1}^{N}\left(\hat{s}_{f}-s_{f}\right)^{2}$.

The extraction performance was evaluated by determining SNR improvement, which is defined as the output SNR minus the input SNR:

$$
S N R_{\text {improve }}=S N R_{\text {out }}-S N R_{\text {in }} .
$$

The correlation coefficient $C_{R}$ was used to evaluate the similarity between the extracted FECG and the original FECG signal. It is important to evaluate the extraction quality of the FECG. $C_{R}$ was calculated using Formula (23):

$$
C_{R}=\frac{\sum_{n=1}^{N} \hat{s}_{f}(n) s_{f}(n)}{\sqrt{\sum_{n=1}^{N}\left(\hat{s}_{f}(n)\right)^{2} \sum_{n=1}^{N}\left(s_{f}(n)\right)^{2}}} .
$$

\section{Experiments and Discussions}

\subsection{Synthetic Data Experiments}

FECG extraction is mainly affected by three factors: the energy ratio of each noise in the mixed signal, the FECG and MECG amplitude ratio (FMAR), and the FECG and MECG heart rate ratio (FMHRR).

\subsubsection{Experiments with the Noise Energy Ratio}

The energy ratio of each noise in the mixed signals affects the extraction of the MECG and identification of the FECG. Since the method in this paper is an adaptive method based on R peak detection, if the FECG is completely contaminated by noise, it will be difficult to identify the R peaks, which may lead to FECG extraction failure. Therefore, in order to verify the effectiveness of the method 
in this paper, the FECG to noise energy ratio (FNR) was taken as the evaluation parameter for the experiments. The FECG to noise energy ratio $(\mathrm{dB})$ is given by Formula (24):

$$
F N R=10 \log _{10}\left(P_{s_{f}} / P_{n}\right)
$$

where $P_{n}=\frac{1}{N} \sum_{n=1}^{N} n^{2}$ is the energy of the noise.

The 5 FECG signals and 21 MECG signals in Table 1 were combined to obtain 105 synthetic signals. All the synthetic signals maintained FECG energy at $1(0 \mathrm{~dB})$ and FMAR at 0.3 . The FMHRR was determined according to the signal combination. The noise energy was changed according to FNR. Figure 7 shows the SNR improvement and correlation coefficient results of adding different energy noises to the 105 synthetic signals using the proposed method. As can be seen, the maximum noise energy is 7.94 times the FECG energy when FNR $=-9$. The minimum noise energy is $1 / 10$ of the FECG energy when $F N R=10$. The red line represents the maximum value of the experimental results of the 105 synthetic signals, while the blue line represents the minimum value. The black line represents the average value. The results show that in the $F N R$ range of $-8 \mathrm{~dB}$ to $10 \mathrm{~dB}$, the SNR improvement $S N R_{\text {improve }}$ increases with increasing $F N R$, but the change is not significant. This is because the increase of FNR indicates a decrease of noise energy, which is conducive to coherent extraction by MECG and FECG. Since the method in this paper does not specifically denoise random noise, the main contribution to SNR improvement comes from MECG elimination. Therefore, in the range of FNR changes, there is no great change in SNR improvement. However, the SNR is improved to a minimum of $12.4 \mathrm{~dB}$, and the average reaches from $17.9 \mathrm{~dB}$ to $19.3 \mathrm{~dB}$, indicating that it has a significant effect for MECG elimination. The correlation coefficient $C_{R}$ increases with increasing FNR. Within the FNR range of $-8 \mathrm{~dB}$ to $10 \mathrm{~dB}$, the minimum $C_{R}$ is 0.69 , the maximum $C_{R}$ is 0.96 , and the average $C_{R}$ is 0.8-0.9. This shows that the FECG extracted successfully using the method in this paper has good similarity with the original FECG.

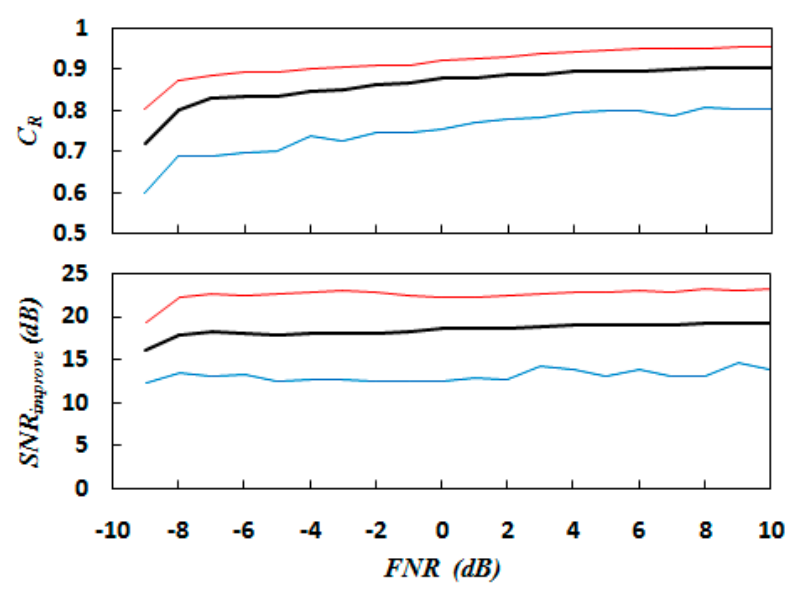

Figure 7. Correlation coefficient and signal-noise ratio (SNR) improvement results against the FECG to noise energy ratio (FNR) using the proposed method for 105 synthetic signals. The red, blue, and black lines present the maximum, minimum, and average values, respectively.

\subsubsection{Experiments with the FMAR}

The 5 FECGs, 21 MECGs, and random white noise in Table 1 were superposed to produce synthetic ECG signals. When synthesising these signals, the FECG energy was maintained at $1(0 \mathrm{~dB})$ and the FNR at $10 \mathrm{~dB}$. The FMAR was used to change the amplitude of the MECG to implement the experiments. Figure 8 shows the SNR improvement and correlation coefficient results of different amplitude ratios for the 105 synthetic signals using the proposed method. The red line represents the maximum value of the experimental results, while the blue line represents the minimum value. 
The black line represents the average value. As can be seen from Figure 8, with increasing FMAR, the $S N R_{\text {improve }}$ decreases correspondingly. This is because with increasing FMAR, the energy of the corresponding MECG will decrease, and the SNR improvement generated by MECG elimination will also decrease accordingly. However, when FMAR increases, the correlation coefficient $C_{R}$ increases, indicating that the quality of the extracted FECG is improving. It is noted that when FMAR is small, the MECG residue is relatively large, which will affect the identification of the FECG. When FMAR is less than 0.1, FECG identification becomes difficult. On the contrary, when FMAR increases, the R peak of the FECG is close to the R peak of the MECG, which will interfere with the identification of the MECG. When FMAR is larger than 0.9, MECG extraction errors will occur. Fortunately, this is rarely the case in actual abdomen mixed signals.

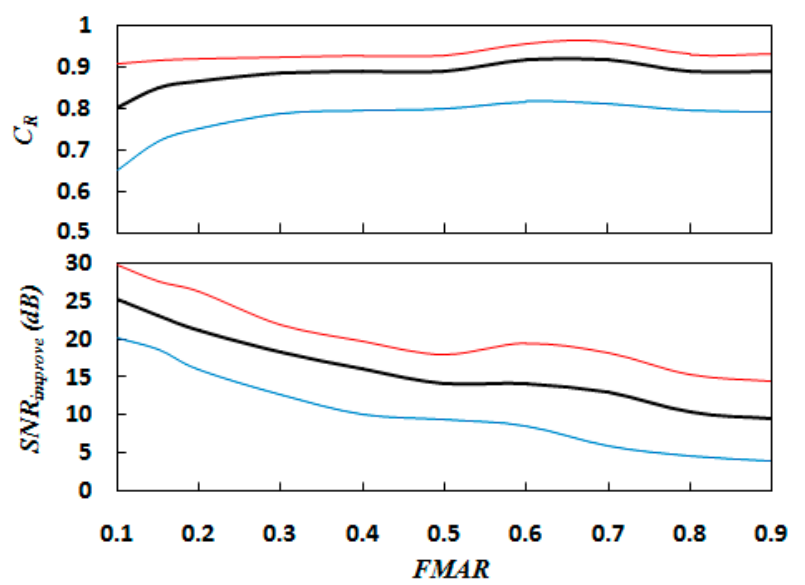

Figure 8. Correlation coefficient and SNR improvement results against the FECG and MECG amplitude ratio (FMAR) using the proposed method for 105 synthetic signals. The red, blue, and black lines present the maximum, minimum, and average values, respectively.

\subsubsection{Effect of Heart Rate Ratio}

According to the statistics of the experimental results in Sections 4.1.1 and 4.1.2, the heart rate ratio range of the 105 mixed signals is 1.2 to 2.8. The experimental statistical results show that the heart rate ratio has no significant effect on the performance of the proposed method, except when the heart rate ratio is equal to 1 and the FECG and MECG are exactly coincident (see Figures 9 and 10).

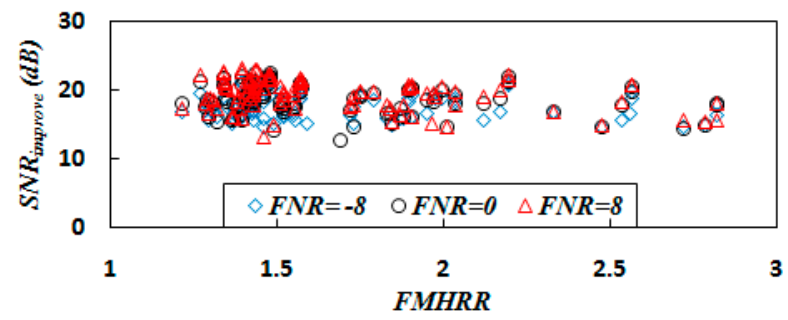

Figure 9. The change of SNR improvement with the FECG and MECG heart rate ratio (FMHRR) when FNR is fixed. 


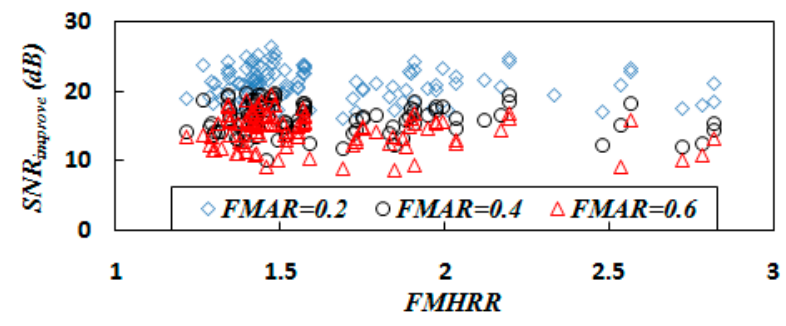

Figure 10. The change of SNR improvement with FMHRR when FMAR is fixed.

\subsection{Real Data Experiments}

In this paper, the DaISy, ADFECGDB, and NIFECGDB databases were selected as real FECG databases.

\subsubsection{DaISy}

Figure 11 displays the extraction results of abdominal mixed signals in Channels $1-5$ of the DaISy database by the proposed method. The results show that the method in this paper has a good extraction effect on DaISy data, and the FECG could be extracted even from ill-formed mixed signals, such as Channel 4 and Channel 5. In addition, it can be seen that the extraction results not only have clear QRS waveforms, but also retain the local variation trends of the $\mathrm{P}$ and $\mathrm{T}$ waves, which is of great significance to the FECG extraction results. Nevertheless, since the method in this paper is fully adaptive, the recognition of $\mathrm{R}$ peaks is an important factor affecting the extraction effect, and individual misjudgement of signals in an ill-conditioned environment may result in the dislocation of $\mathrm{R}$ peaks in the extraction results, such as from $2 \mathrm{~s}$ to $3 \mathrm{~s}$ of Channel 4 and Channel 5 . However, individual misjudgements do not affect the FECG analysis or heart rate calculations.

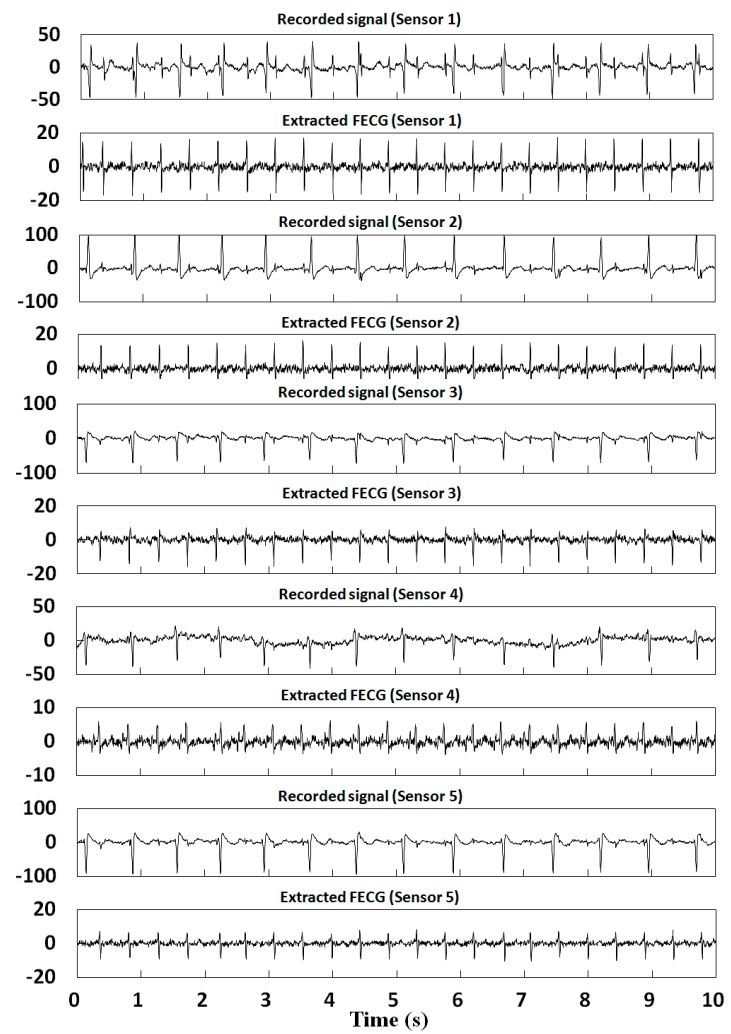

Figure 11. Extraction results from abdominal signals for Channels $1-5$ in the DaISy database. 


\subsubsection{ADFECGDB}

In this section, $10 \mathrm{~s}$ of time was selected for the FECG extraction experiment. Table 2 shows the correlation coefficients of the FECG extraction results by our method. Figures 12-16 show the experimental results of all 20 abdominal channels of the ADFECGDB database. The results show that, except for Channel 1 of r04, Channel 1 of r07, and Channel 3 of r10 having no observable FECG signals, FECGs were well extracted from all other channels with good correlation coefficients. The FECGs extracted successfully by our method have complete QRS waveforms and accurate R peak positions, $\mathrm{P}$ waves and $\mathrm{T}$ waves are clear, and the trend is reasonable.

Table 2. Correlation coefficients between the extracted FECGs and the clean scalp FECGs.

\begin{tabular}{ccccc}
\hline & ch1 & ch2 & ch3 & ch4 \\
\hline r01 & 0.798 & 0.764 & 0.756 & 0.847 \\
\hline r04 & & 0.795 & 0.785 & 0.808 \\
\hline r07 & & 0.821 & 0.767 & 0.802 \\
\hline r08 & 0.737 & 0.659 & 0.701 & 0.703 \\
\hline r10 & 0.781 & 0.742 & & 0.571 \\
\hline
\end{tabular}

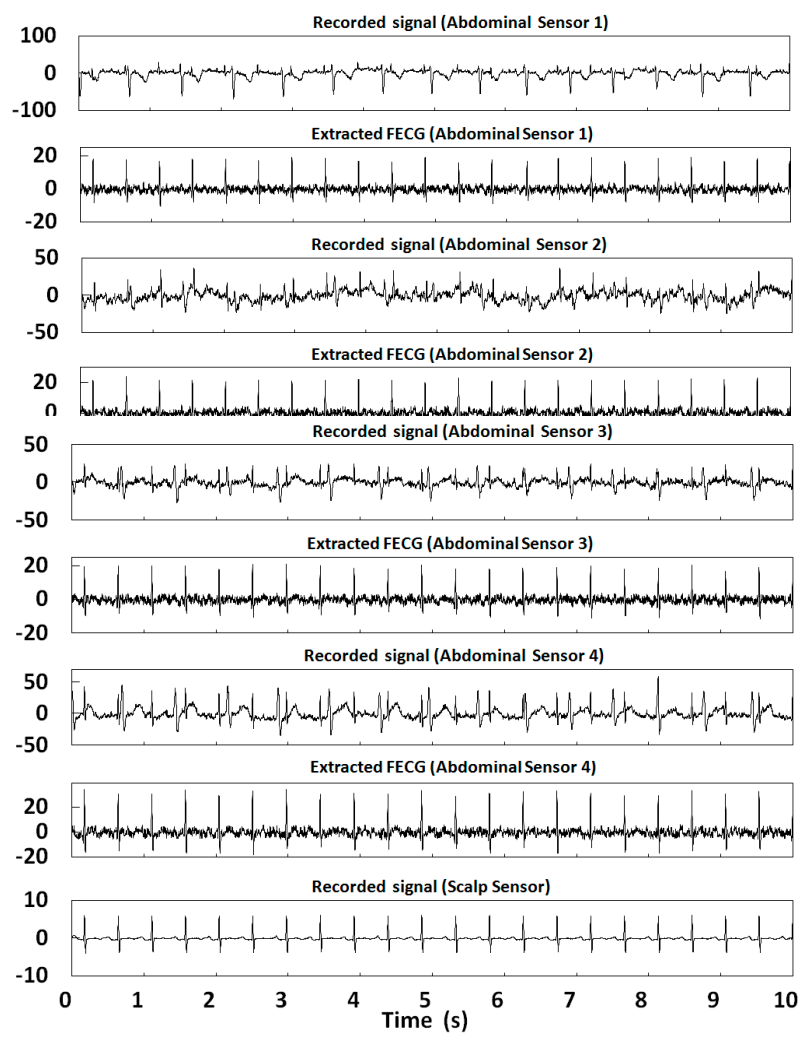

Figure 12. Extraction results for r01 in the ADFECGDB database. 


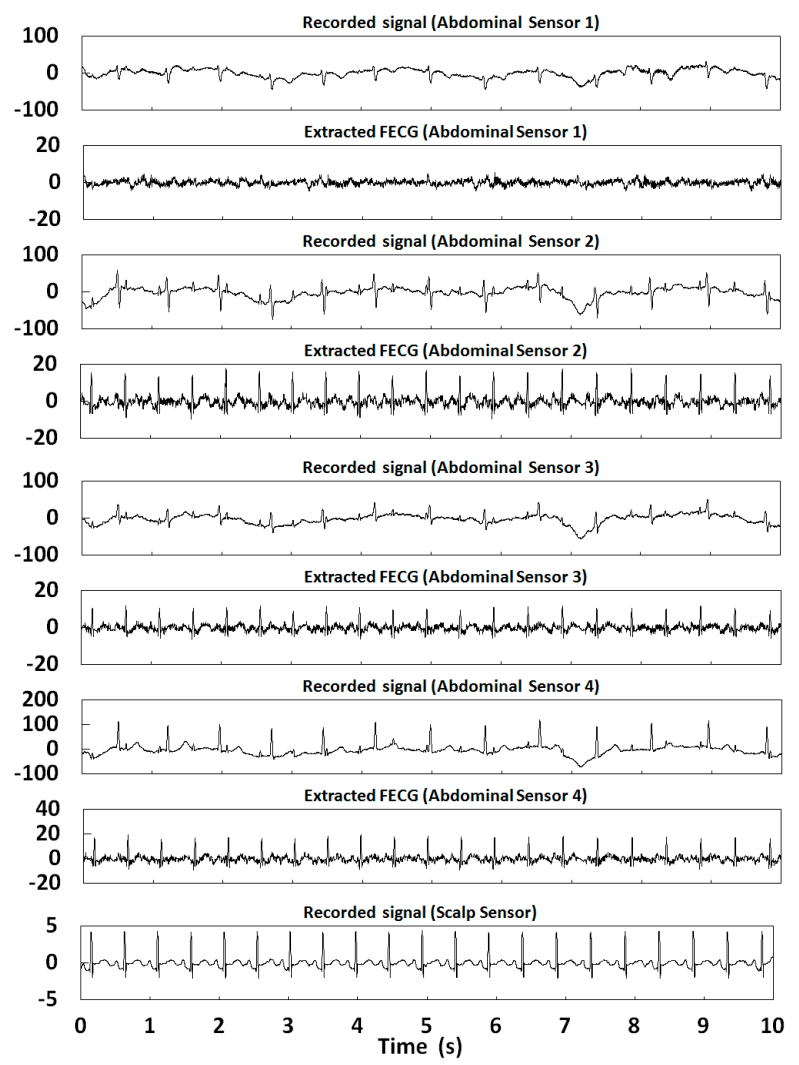

Figure 13. Extraction results for r04 in the ADFECGDB database.

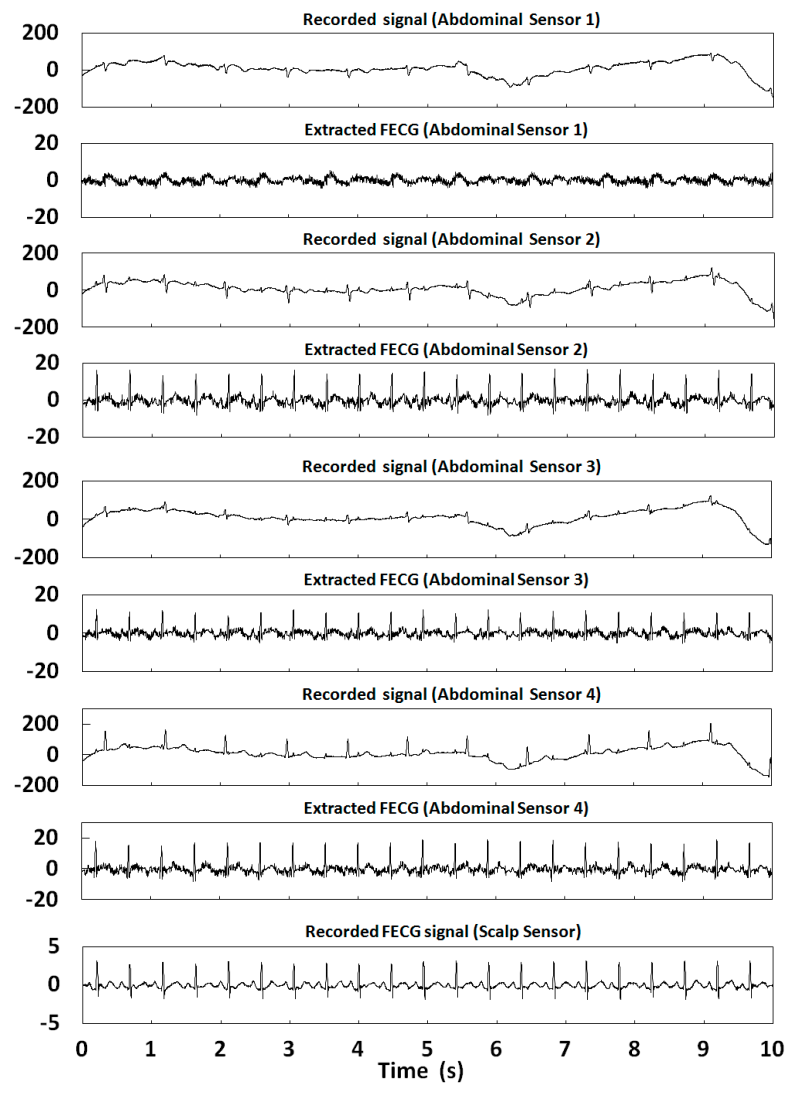

Figure 14. Extraction results for r07 in the ADFECGDB database. 


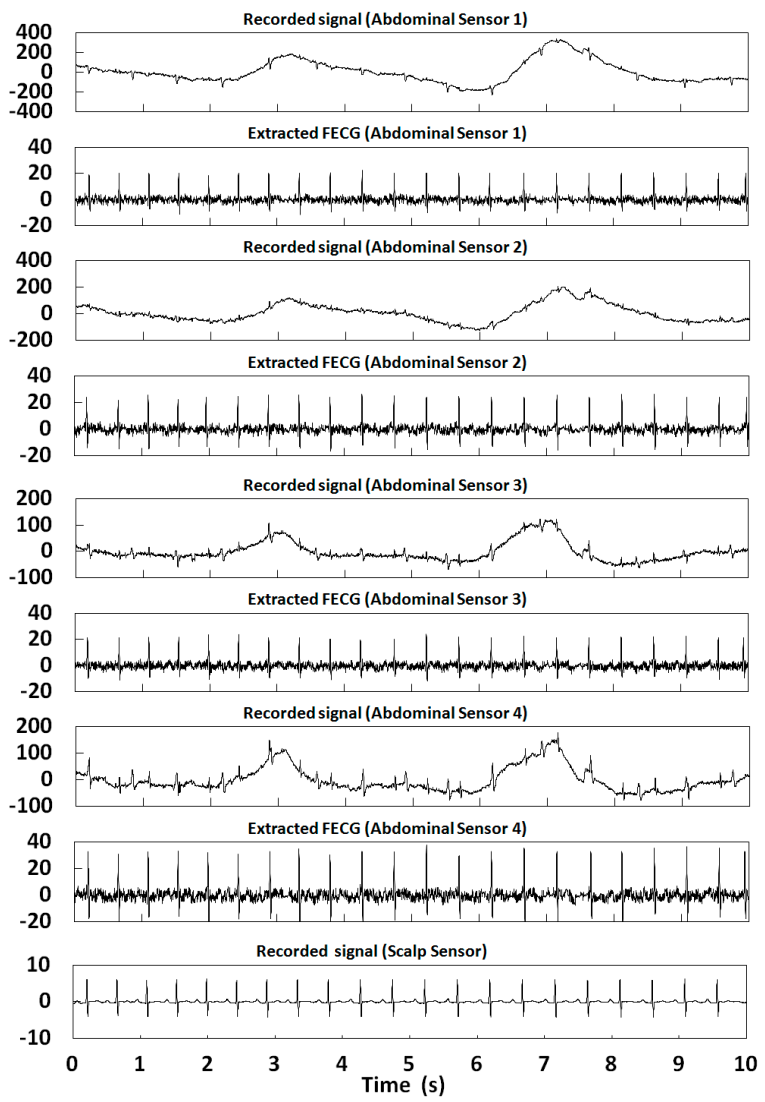

Figure 15. Extraction results for r08 in the ADFECGDB database.

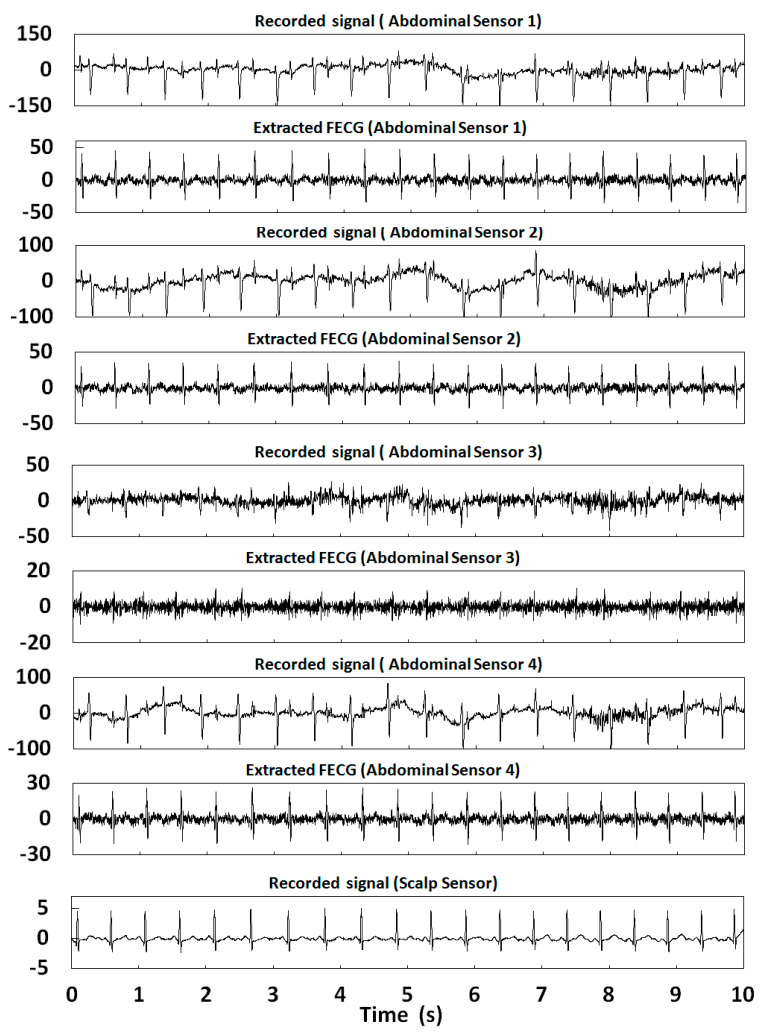

Figure 16. Extraction results for $r 10$ in the ADFECGDB database. 


\subsubsection{NIFECGDB}

In order to further verify the effectiveness of FECG extraction at different pregnancy stages and channels by the proposed method, eight records were selected for experiments. These were ecgca473 Channel 5, ecgca585 Channel 6, ecgca649 Channel 3, ecgca748 Channel 5, ecgca771 Channel 3, ecgca840 Channel 4, ecgca886 Channel 3, and ecgca902 Channel 5. The experimental results are shown in Figure 17.
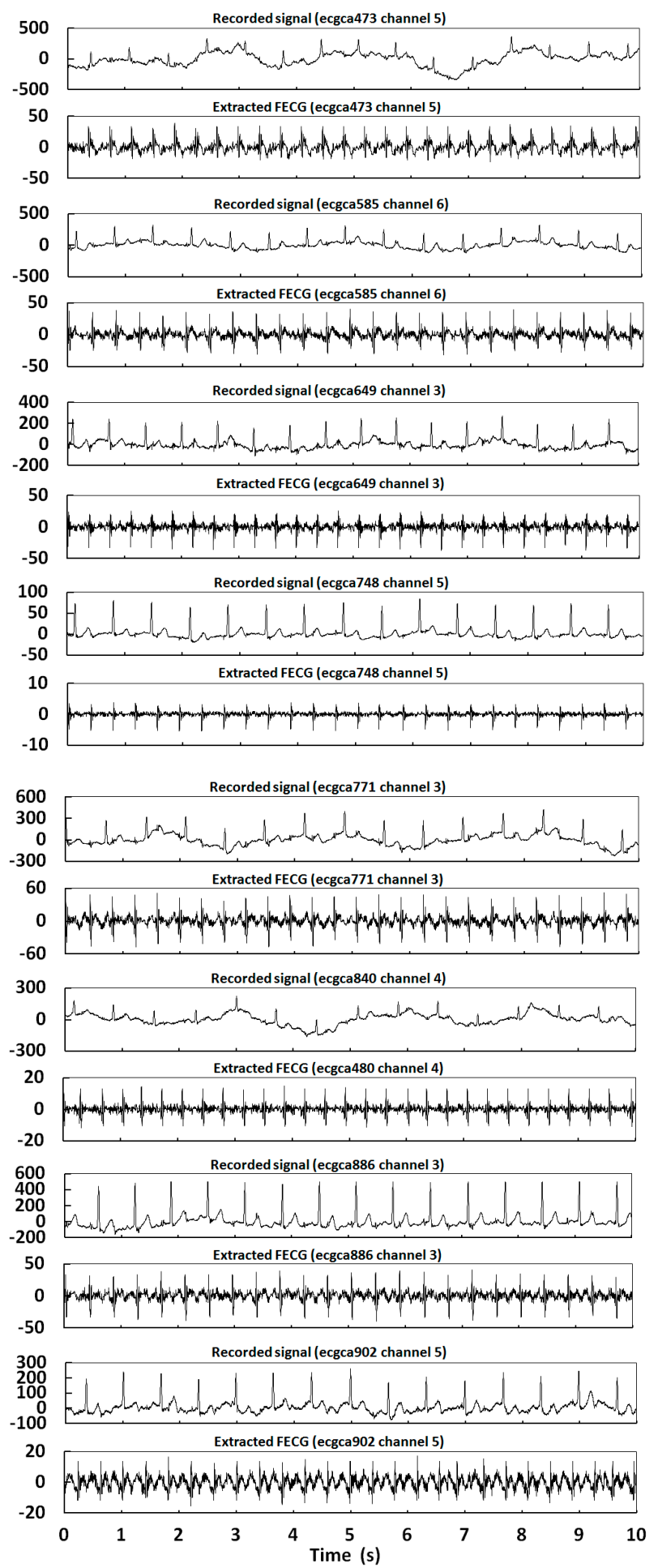

Figure 17. Extraction results for eight records in the NIFECGDB database. 


\section{Conclusions}

In this paper, a single-channel FECG extraction method based on an instantaneous correlation coefficient and simplified coherent averaging method is proposed. The method is a completely adaptive extraction method based on the characteristics of the signals themselves, unlike the existing methods. By using the standard heart beat model for relevant calculations, the positions of the R peaks are highlighted effectively, and the identification accuracy of the $\mathrm{R}$ peaks is improved. By calculating the average waveform of all of the heart beats, the proposed simplified coherent averaging method can obtain the stationary information and then retain the nonstationary and nonlinear information in the residue signals. Thus, FECG information is not lost when the coherent averaging MECG is eliminated. The variance of the coherent averaging FECG can be used to identify the amplitude of the FECG nonstationary information, which is used as the threshold to eliminate the MECG residues. In this way, the FECG's useful components and nonstationary information in the remaining signals can be effectively retained, so that the nonlinear and nonstationary characteristics of the final extracted FECG can be subsequently recovered. The validity of the proposed method was verified by way of experiments using synthetic data and real database data. Through the synthetic data experiment established in this paper, it was shown that the method in this paper can effectively extract FECGs when the FECG to MECG amplitude ratio is within the range of 0.1 to 0.9 . In other cases, the success rate of extraction is reduced. This is because the MECG residue is relatively large when the FECG to MECG amplitude ratio is less than 0.1, which interferes with FECG identification. When it is larger than 0.9 , the R peak sizes of the FECG and MECG are similar, which affects the MECG R peak detection. When the energy ratio of FECG to noise is greater than $-8 \mathrm{~dB}$ and the heart rate ratio of FECG to MECG is greater than 1.1, changes in the noise energy and heart rate ratio have no significant effect on the performance of the method proposed in this paper. The synthetic data and real database experiment results show that FECGs extracted by this method are of high quality according to their SNR improvement, correlation coefficient, and signal waveforms. The QRS complex wave of the FECG is clear. The form of the P and T waves is reasonably enhanced. The waveform amplitude and each interval have no obvious distortion, and the nonlinear and nonstationary characteristics can be fully retained.

Author Contributions: Data curation, M.Z.; Formal analysis, M.Z.; Methodology, M.Z.; Supervision, G.W.; Writing - original draft, M.Z.; Writing_-review and editing, M.Z. and G.W. All authors have read and agreed to the published version of the manuscript.

Funding: This research received no external funding.

Conflicts of Interest: The authors declare no conflict of interest.

\section{References}

1. Jenkins, H.M. Technical progress in fetal electrocardiography-A review. J. Perinat. Med. 1986, 14, 365-370. [PubMed]

2. Jenkins, H.M. Thirty years of electronic intrapartum fetal heart rate monitoring: Discussion paper. J. R. Soc. Med. 1989, 82, 210-214. [CrossRef] [PubMed]

3. Sameni, R.; Clifford, G.D. A review of fetal ECG signal processing; issues and promising directions. Open Pacing Electrophysiol. Ther. J. 2010, 3, 4-20. [CrossRef] [PubMed]

4. Han, G.; Lin, B.; Xu, Z. Electrocardiogram signal denoising based on empirical mode decomposition technique: An overview. J. Instrum. 2017, 12, P03010. [CrossRef]

5. Awal, M.A.; Sheikh, S.M.; Mohiuddin, A.; Mohd, A.R. An adaptive level dependent wavelet thresholding for ECG denoising. Biocybern. Biomed. Eng. 2014, 34, 238-249. [CrossRef]

6. Liu, H.; Chen, D.; Sun, G. Detection of Fetal ECG R Wave from Single-Lead Abdominal ECG Using a Combination of RR Time-Series Smoothing and Template-Matching Approach. IEEE Access 2019, 7, 66633-66643. [CrossRef] 
7. Cerutti, S.; Baselli, G.B.; Civardi, S.; Ferrazzi, E.; Marconi, A.M.; Pagani, M.; Pardi, G. Variability analysis of fetal heart rate signals as obtained from abdominal electrocardiographic recordings. J. Perinat. Med. 1986, 14, 445-452. [CrossRef]

8. Martens, S.M.M.; Rabotti, C.; Mischi, M.; Sluijter, R.J. A Robust Fetal ECG Detection Method for Abdominal Recordings. Physiol. Meas. 2007, 28, 373-388. [CrossRef]

9. Ungureanu, M.; Bergmans, J.W.M.; Oei, S.G.; Strungaru, R. Fetal ECG extraction during labor using an adaptive maternal beat subtraction technique. Biomed. Tech. 2007, 52, 56-60. [CrossRef]

10. Behar, J.; Johnson, A.; Clifford, G.D.; Oster, J. A comparison of single channel fetal ECG extraction methods. Ann. Biomed. Eng. 2014, 42, 1340-1353.

11. Puthusserypady, S. Extraction of fetal electrocardiogram using H-infinity adaptive algorithms. Med. Biol. Eng. Comput. 2007, 45, 927-937. [CrossRef] [PubMed]

12. Kiruthiga, G.; Sharmil, A.A.; Mahalakshmi, P. Enhancement of fetal ECG signal using adaptive noise cancellation. Int. J. Pharma. Biol. Sci. 2015, 6, 943-950.

13. Wu, S.; Shen, Y.; Zhou, Z.; Lin, L.; Zeng, Y.; Gao, X. Research of fetal ECG extraction using wavelet analysis and adaptive filtering. Comput. Biol. Med. 2013, 43, 1622-1627. [CrossRef] [PubMed]

14. Khamene, A.H.; Negahdaripoure, S. A new method for the extraction of fetal ECG from the composite abdominal signal. IEEE Trans. Biomed. Eng. 2000, 47, 507-516. [CrossRef]

15. Karvounis, E.C.; Tsipouras, M.G.; Fotiadis, D.I.; Naka, K.K. An automated methodology for fetal heartrate extraction from the abdominal electrocardiogram. IEEE Trans. Inf. Technol. Biomed. 2007, 11, 628-638. [CrossRef]

16. Castillo, E.; Morales, D.P.; Botella, G.; Garcia, A.; Parrilla, L.; Palma, A.J. Efficient wavelet-based ECG processing for single-lead FHR extraction. Digit. Signal Process. 2013, 23, 1897-1909. [CrossRef]

17. Almeida, R.; Goncalves, H.; Bernardes, J.; Rocha, A.P. Fetal QRS detection and heart rate estimation: A wavelet-based approach. Physiol. Meas. 2014, 35, 1723-1735. [CrossRef]

18. Lamesgin, G.; Kassaw, Y.; Assefa, D. Extraction of Fetal ECG from Abdominal ECG and Heart Rate Variability Analysis. Adv. Intell. Syst. Comput. 2015, 334, 147-161.

19. Lee, K.; Lee, B. Sequential Total Variation Denoising for the Extraction of Fetal ECG from Single-Channel Maternal Abdominal ECG. Sensors 2016, 16, 1020. [CrossRef]

20. Panigraphy, D.; Sahu, P.K. Extraction of fetal ECG by extended state Kalman filtering and adaptive neuro-fuzzy inference system based on single channel. Sadhana 2015, 40, 1091-1104. [CrossRef]

21. Kanjilal, P.P.; Palit, S.; Saha, G. Fetal ECG extraction from single-channel maternal ECG using singular value decomposition. IEEE Trans. Biomed. Eng. 1997, 44, 51-59. [CrossRef]

22. Zhang, Y.; Yu, S. Single-lead noninvasive fetal ECG extraction by means of combining clustering and principal components analysis. Med. Biol. Eng. Comput. 2020, 58, 419-432. [CrossRef]

23. Richter, M.; Schreiber, T.; Kaplan, D.T. Fetal ECG extraction with nonlinear state-space projections. IEEE Trans. Biomed. Eng. 1998, 45, 133-137. [CrossRef] [PubMed]

24. Kotas, M.; Jezewski, J.; Matonia, A.; Kupka, T. Towards noise immune detection of fetal QRS complexes. Comput. Methods Programs Biomed. 2010, 97, 241-256. [CrossRef] [PubMed]

25. Nagendra, H.; Mukherjee, S.; Kumar, V. Application of wavelet techniques in ECG signal processing: An overview. Int. J. Eng. Sci. Tech. 2011, 3, 7432.

26. Van Mieghem, T.; DeKoninck, P.; Steenhaut, P.; Deprest, J. Methods for Prenatal Assessment of Fetal Cardiac Function. Prenat. Diagn. 2009, 29, 1193-1203. [CrossRef] [PubMed]

27. DuBose, T.J.; Cunyus, J.A.; Johnson, L.F. Embryonic heart rate and age. J. Diagnost. Med. Sonogr. 1990, 6, 151-157. [CrossRef]

28. Mohammad, N.; Bertrand, R.; Christian, J. Fetal ECG Extraction by Extended State Kalman Filtering Based on Single-Channel Recordings. IEEE Trans. Biomed. Eng. 2013, 60, 1345-1352.

29. McSharry, P.E.; Clifford, G.D. A Dynamical Model for Generating Synthetic Electrocardiogram Signals. IEEE Trans. Biomed. Eng. 2003, 50, 289-294. [CrossRef]

30. Sameni, R.; Clifford, G.D.; Jutten, C.; Shamsollahi, M.B. Multichannel ECG and Noise Modeling: Application to Maternal and Fetal ECG Signals. EURASIP J. Adv. Signal Process. 2007, 1, 043407. [CrossRef]

31. Jezewski, J.; Matonia, A.; Kupka, T.; Roj, D.; Czabanski, R. Determination of the fetal heart rate from abdominal signals: Evaluation of beat-to-beat accuracy in relation to the direct fetal electrocardiogram. Biomed. Eng. 2012, 57, 383-394. [CrossRef] [PubMed] 
32. The MIT-BIH Non-Invasive Fetal Electrocardiogram Database. Available online: http://www.phsionet.org/ pn3/nifecgdb (accessed on 6 September 2007).

33. Moor, B.D.; Gersem, P.D.; Schutter, B.D.; Favoreel, W. DAISY: A database for identification of systems. Journal A 1997, 38, 4-5.

34. Moody, G.B.; Mark, R.G. The MIT-BIH Arrhythmia Database on CD-ROM and software for use with it. Proc. Comput. Cardiol. 1990, 185-188. [CrossRef]

(C) 2020 by the authors. Licensee MDPI, Basel, Switzerland. This article is an open access article distributed under the terms and conditions of the Creative Commons Attribution (CC BY) license (http://creativecommons.org/licenses/by/4.0/). 